\section{Duração da amamentação após a introdução de outro leite: seguimento de coorte de crianças nascidas em um hospital universitário em São Paulo}

\section{Duration of breastfeeding after the introduction of another milk: a cohort follow-up of children born in a university hospital in São Paulo}

\author{
Milena Baptista Bueno \\ Departamento de Nutrição \\ Faculdade de Saúde Pública \\ Universidade de São Paulo \\ Correspondência para/Correspondence to: \\ Av. Dr. Arnaldo, 715 - Cerqueira César \\ 01246-904 - São Paulo - SP \\ mibueno@usp.br \\ José Maria Pacheco Souza \\ Departamento de Epidemiologia \\ Faculdade de Saúde Pública \\ Universidade de São Paulo \\ Suzana Maria Rebêlo Sampaio da Paz \\ Departamento de Nutrição \\ Faculdade de Saúde Pública \\ Universidade de São Paulo \\ Sonia Buongermino de Souza \\ Departamento de Nutrição \\ Faculdade de Saúde Pública \\ Universidade de São Paulo \\ Priscila Po Yee Cheung \\ Departamento de Epidemiologia \\ Faculdade de Saúde Pública \\ Universidade de São Paulo \\ Rosangela Aparecida Augusto \\ Departamento de Epidemiologia \\ Faculdade de Saúde Pública \\ Universidade de São Paulo

\section{Auxílio financeiro} \\ Fundação de Amparo à Pesquisa do Estado de São Paulo - FAPESP (Processo \\ No 96/06073-2)
}

\section{Resumo}

O objetivo deste trabalho foi estudar a duração do aleitamento materno após a introdução de outro leite na alimentação infantil. Foi analisada uma coorte de 450 crianças selecionadas no hospital universitário de São Paulo na ocasião do parto, entre 1998 e 1999. Informações diárias sobre a alimentação da criança foram obtidas a partir de registro feito pela mãe. Para análise dos dados foi utilizada a técnica de análise de sobrevida, atuarial e Kaplan-Meier, e o modelo de Cox. Cinqüenta e quatro crianças (12,0\%) não chegaram a receber leite não materno durante o tempo de observação, 193 (43,0\%) tiveram a introdução de outro leite até sessenta dias de idade (grupo 1), 151 (33,5\%) receberam leite não materno pela primeira vez entre sessenta e um e cento e oitenta dias (grupo 2) e 52 (11,5\%) passaram a receber leite não materno depois de cento e oitenta dias (grupo 3). Os tempos medianos de duração da amamentação para os três grupos, após a introdução do leite não materno, foram, respectivamente, 76, 120 e 176 dias. Tomando como categoria basal o primeiro grupo, a razão de hazards do segundo grupo foi 0,73 (IC: $0,57-0,94$ ) e a do terceiro foi 0,43 (IC: 0,26-0,72). Concluiu-se que quanto mais tarde é introduzido o outro leite, por mais tempo a mãe tende a amamentar, e as mães que desejam prolongar a amamentação retardam a introdução de outro leite e, após a introdução, mantêm pelo maior tempo possível a concomitância do outro leite com o leite materno.

Palavras-chave: Amamentação. Leite não materno. Desmame. Coorte. 
Abstract

The duration of breastfeeding after the introduction of another milk in child feeding was studied, by following a cohort of mothers and their infants during one year. Daily information about breastfeeding, introduction of non maternal milk, besides other events concerning the child, were registered by the mother in a specially formatted sheet, with a 60-day "capacity". Trained interviewers visited the mothers at infant ages of 15 , $30,60,90,180,270$ and 360 days to return and replace the sheets. Four hundred and fifty children provided valid information to be included in the analysis. Survival analysis techniques were used to describe and to compare the duration of breastfeeding after the introduction of non maternal milk. Fifty four $(12.0 \%)$ infants never received non maternal milk during the observation period; 193 (43,0\%) received non maternal milk within the first sixty days of birth (group 1), 151 (33.5\%) started receiving non maternal milk between sixty one and one hundred and eighty days (group 2) and 52 (11,5\%) thereafter (group 3). The median times of duration of breastfeeding after the introduction were, respectively, 76,120 and 176 days. Taking the first group as the base category, the hazards ratio of the second group was 0.73 (IC: 0.57 0.94 ) and 0.43 (IC: $0.26-0.72$ ) for the third group. It was concluded that the earlier the introduction of non maternal milk the faster the process of weaning, and that mothers intending to breastfeed their children for a large period do it delaying the introduction of non maternal milk, and maintaining both breastfeeding and non maternal milk after the introduction, for as long as possible.

Keywords: Breastfeeding. Non-maternal milk. Weaning. Cohort.

\section{Introdução}

A Organização Mundial de Saúde recomenda que o aleitamento materno exclusivo seja mantido até os 6 meses de idade da criança, a partir de quando é necessária a introdução de alimentos complementares, em conjunto com a continuação da amamentação ${ }^{1}$. Por alimento complementar entende-se qualquer alimento nutritivo, sólido ou líquido, diferente do leite materno ${ }^{2}$.

Os benefícios do aleitamento materno, especialmente do aleitamento materno exclusivo, estão bem estabelecidos ${ }^{1}$, principalmente em países subdesenvolvidos, onde a introdução precoce de outro leite pode aumentar o risco de morbidade e de desnutrição, devido à contaminação da água e diluição excessiva do leite ${ }^{3}$.

Eventos que se manifestam durante a infância ou adolescência, tais como diabetes melitus tipo $1^{4-6}$, obesidade ${ }^{7}$, asma ${ }^{8}$ e alergia $^{9}$, podem estar associados à introdução precoce de leite não materno.

Os fatores determinantes da interrupção precoce da amamentação têm sido objeto de investigação; nível de escolaridade da mãe e sua inserção no mercado de trabalho, condições socioeconômicas, problemas de saúde da mãe ou da criança, uso de bicos artificiais ou chupetas e atuação dos serviços de saúde são algumas das variáveis estudadas ${ }^{10-12}$.

A introdução de alimentos complementares diminui a duração do aleitamento materno, principalmente se esta introdução ocorrer precocemente $\mathrm{e}^{10-12}$. Quando um alimento sólido é introduzido, há a necessidade de a criança se adaptar à nova textura e à sua administração, ao contrário do leite não materno, fluido ou em pó, que geralmente é oferecido em mamadeiras, facilitando o seu consumo, o que leva a uma redução mais rápida da amamentação, quando comparada com a introdução de sólidos ${ }^{13}$.

No Brasil, um dos fatores responsáveis pelo declínio da amamentação, juntamente com a urbanização e a inserção da mulher no mercado de trabalho, foi a entrada no país da indústria de leite em pó e a propaganda destes produtos por meio de estraté- 
gias de marketing que visavam atingir os profissionais de saúde, além do público em geral $^{14,15}$. A partir da década de 70, o governo brasileiro inicia o Programa Nacional de Incentivo ao Aleitamento Materno ${ }^{16}$.

A introdução de leite não materno talvez seja um dos principais iniciadores e aceleradores (fator de risco) do processo de desmame que leva ao fim do aleitamento materno, independentemente de outros alimentos $^{17}$. O presente trabalho descreve a duração do processo de desmame somente como função da época de introdução de outro leite, independentemente do consumo de outros alimentos.

\section{Materiais e métodos}

Este é parte de um estudo de coorte prospectivo de crianças nascidas sem intercorrências no Hospital Universitário da Universidade de São Paulo (HU), com o objetivo, entre outros, de estudar a alimentação de crianças no primeiro ano de vida.

No HU nascem, em média, sete crianças por dia, cujas mães podem ser classificadas como da comunidade USP (alunas, funcionárias docentes e não docentes, dependentes de funcionários) e da comunidade (residentes em região delimitada próxima ao hospital). O HU tem programa de alojamento conjunto, porém não tem programa rotineiro de puericultura; as mães são orientadas a fazerem este acompanhamento nos centros de saúde.

Devido à factibilidade operacional, foram selecionadas quatro crianças por dia, em ordem de nascimento no dia anterior, a partir de outubro de 1998. Não foram incluídas crianças com malformação congênita, nascidas de parto gemelar, que necessitassem de internação hospitalar por um período superior a 5 dias, com morte materna, ou com a mãe necessitando internação por mais de 5 dias.

Inicialmente, 609 mães aceitaram participar da pesquisa. Foram excluídas 21 crianças por não residirem na região de abrangência da pesquisa (residentes em outro município). Na primeira visita, 506 crianças foram identificadas nas residências, constituindo-se no real tamanho inicial da coorte estudada. Por tratar-se de processo dinâmico, ocorreram desistências e perdas no período, conforme mostra a tabela 1. É importante notar que foram aproveitadas informações parciais das 123 crianças perdidas ao longo de um ano, após a primeira visita domiciliar. O principal motivo destas perdas foi mudança de endereço $(83,7 \%)$.

\section{Tabela 1 - Dinâmica da cohort.}

Table 1 - Dynamics of the cohort

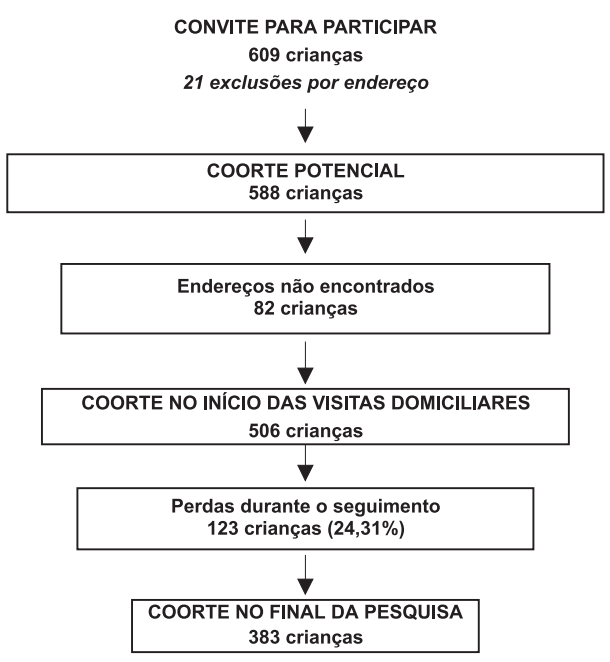

Não houve diferença estatisticamente significante entre as crianças que completaram o seguimento (383), as que não foram localizadas na primeira visita domiciliar (82) e aquelas que iniciaram o seguimento porém não o finalizaram (123). As características analisadas foram: sexo ( $\mathrm{p}=0,97)$, raça $(\mathrm{p}=0,17)$, ordem de nascimento $(\mathrm{p}=0,31)$, peso ao nascer $(\mathrm{p}=0,07)$, comprimento ao nascer $(\mathrm{p}=0,34)$, perímetro cefálico ao nascer $(\mathrm{p}=0,16)$, tempo de gestação $(\mathrm{p}=0,77)$, ganho de peso na gestação $(\mathrm{p}=0,53)$, número de consultas pré-natal $(\mathrm{p}=0,44)$, tipo de parto $(\mathrm{p}=0,65)$, número de gestações anteriores ( $\mathrm{p}=0,43)$, hábito de fumar materno durante a gestação $(\mathrm{p}=0,24)$, condição marital $(\mathrm{p}=0,83)$, idade materna $(\mathrm{p}=0,41)$ e escolaridade materna $(\mathrm{p}=0,54)$.

O presente trabalho abrange 450 crianças que devolveram ao menos a primeira 
planilha contendo informações sobre alimentação, com o registro dos primeiros 60 dias de vida da criança.

As entrevistas domiciliares foram realizadas aos 15, 30, 60, 90, 180, 270 e 360 dias de idade da criança. No recrutamento, ainda no hospital, cada mãe recebeu um questionário desenhado como planilha para leitura ótica, onde deveria marcar, dia a dia, os eventos relativos à alimentação do filho até a idade de 60 dias; nas visitas domiciliares, as planilhas preenchidas eram recolhidas e novas eram entregues, cada uma para registro de 60 dias.

Cada folha da planilha tinha uma coluna de alvéolos para cada dia do ano, 60 colunas por folha. As linhas dos alvéolos correspondiam a diversos alimentos; no caso do presente trabalho foram utilizadas as linhas de registro de leite de peito, leite fluido, leite em pó e leite de soja. As mães foram orientadas a "pintar" o(s) alvéolo(s) correspondente(s) à combinação dia/tipo de leite(s) consumido(s). Observando a seqüência de marcação, foi possível estabelecer, para cada criança, até que idade ela recebeu leite de peito e com que idade foi introduzido outro tipo de leite que não o materno; a variável número de dias que a criança leva para deixar de receber leite de peito após a introdução de outro leite é a diferença das duas idades. Utilizou-se a abordagem de análise de sobrevida, técnicas atuarial e Kaplan-Meier e modelo de Cox, para descrição e análise dos resultados. Para isso, as crianças que não completaram o seguimento de um ano foram censuradas na última informação sobre a alimentação ${ }^{18}$.

Usando a técnica atuarial, são apresentadas as proporções de crianças ainda sem introdução de outro leite que não o materno, ou seja, recebem leite materno complementado ou não por água, chá e/ou alimentos sólidos. As proporções de crianças ainda em amamentação após a introdução de outro leite, segundo o tempo em dias depois da introdução, são descritas pela técnica Kaplan-Meier. As crianças foram classificadas segundo a idade de introdução do outro leite: até 60 dias (grupo 1), entre 61 e 180 (grupo 2), depois de 180 dias (grupo 3). Usando o primeiro grupo como basal, aplicou-se o modelo de Cox para a obtenção das razões de hazards dos outros dois grupos em relação ao basal. A variável idade de introdução do outro leite foi, ainda, testada segundo seus valores originais.

\section{Resultados}

Das 450 crianças estudadas, $52 \%$ pertenciam ao sexo masculino, $34,7 \%$ residiam em favelas e $58,7 \%$ eram da raça branca. Quanto às mães destas crianças, $44,6 \%$ eram primíparas, $54,4 \%$ estudaram até o ensino fundamental, $5,5 \%$ iniciaram o ensino superior, 55,5\% eram menores de 25 anos, $81,6 \%$ declararam ser casadas e $20,2 \%$ eram fumantes. O peso médio das crianças ao nascer foi de 3.253 gramas (desvio padrão=409,84), com valor mínimo de 2.200 gramas e valor máximo de 4.600 gramas.

Aos seis meses de vida, $98,4 \%$ das crianças já estavam consumindo leite, água, chá e/ou outros alimentos sólidos. A mediana do aleitamento materno exclusivo foi 23 dias e do aleitamento materno, 205.

Das 450 crianças, 54 (12\%) não receberam outro tipo de leite durante o tempo de sua observação, 193 (43\%) tiveram a introdução de outro leite até sessenta dias, 151 (34\%) receberam leite não materno pela primeira vez entre sessenta e um e cento e oitenta dias e $52(12 \%)$ passaram a receber leite não materno depois de cento e oitenta dias.

A Figura 1, que leva em consideração as censuras, mostra como a proporção de crianças ainda sem receber leite não materno vai caindo; aos 6 meses de idade, $84 \%$ das crianças já consomem outro leite e ao final do primeiro ano, $94 \%$. Usando dados não agrupados, a idade mediana, na qual 50\% das crianças já consomem outro leite, é 62 dias.

A Figura 2 ilustra o processo de desmame a partir da introdução do leite não materno; após 91 dias de sua introdução, 50\% das crianças já abandonaram totalmente a amamentação. 


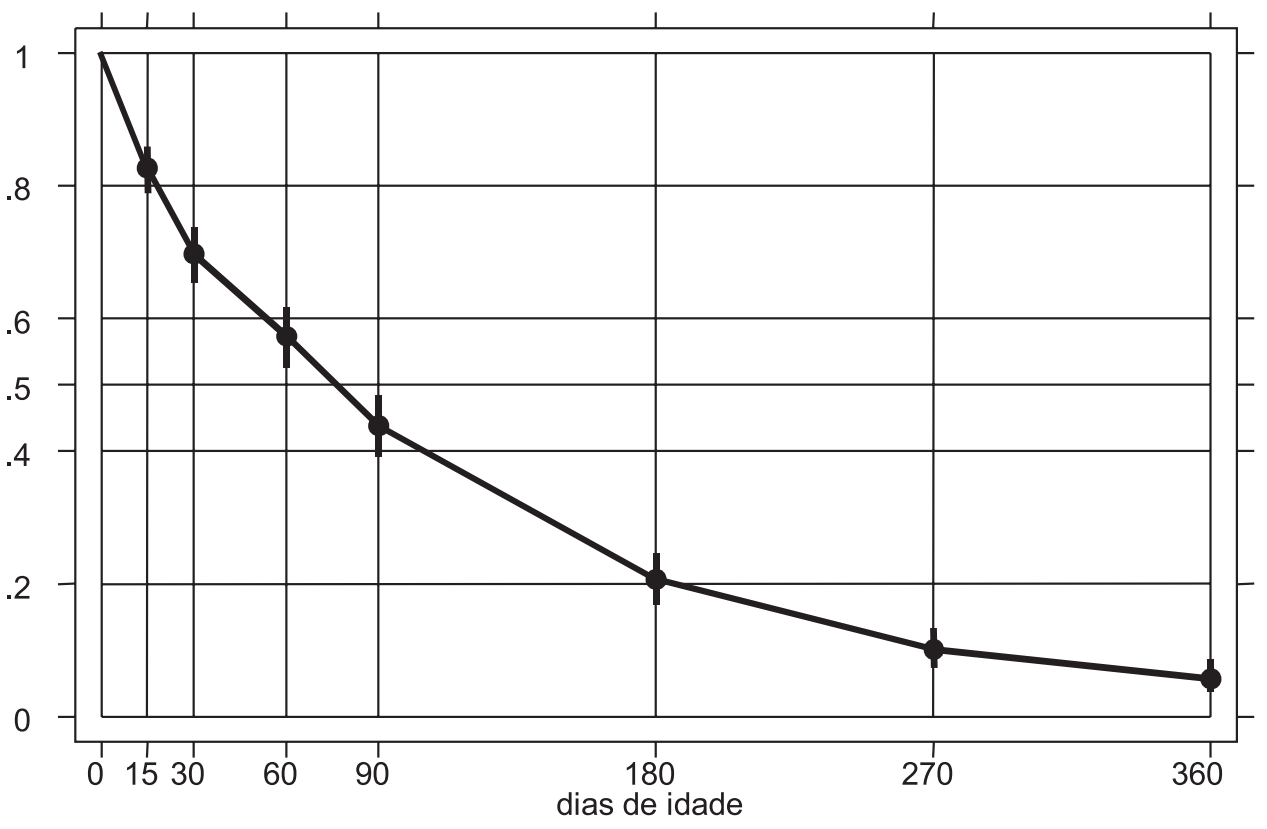

Figura 1 - Proporção de crianças que não consomem leite não materno ao longo do primeiro ano de vida.

Figure 1 - Proportion of children without the introduction of non-maternal milk during the first year of life.

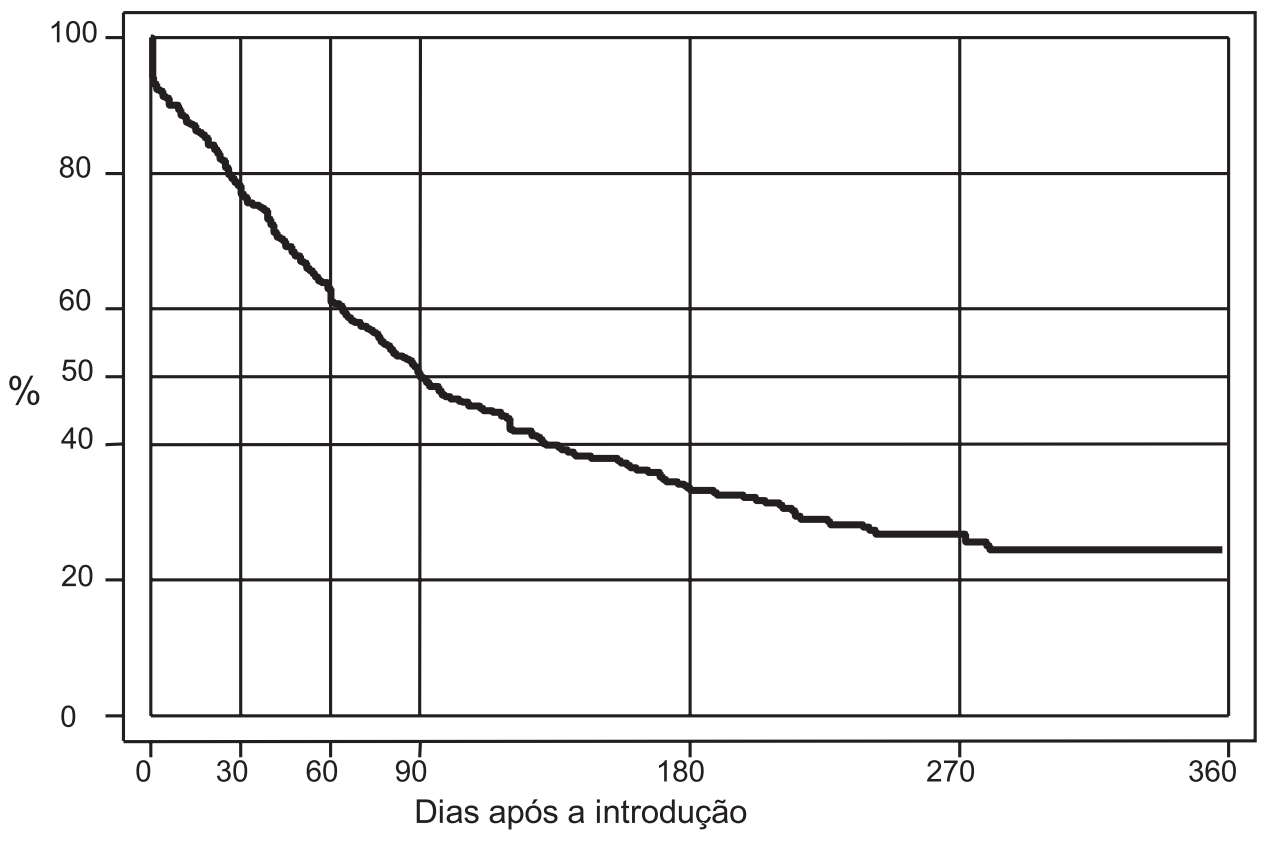

Figura 2 - Duração da amamentação em dias a partir da data da introdução de leite não materno.

Figure 2 - Proportion of children still breastfeeding, after the introduction of non-maternal milk. 
A curva inferior da Figura 3 apresenta a duração da amamentação após a introdução do leite não materno em crianças que tiveram esta introdução em alguma época até 60 dias, caracterizando o desmame mais acelerado entre os 3 grupos; a curva intermediária corresponde ao grupo cuja introdução do outro leite foi entre 61 e 180 dias; a curva superior, com desmame mais lento, é de crianças cuja introdução do outro leite foi feita a partir de 181 dias. Os tempos medianos de duração da amamentação, após a introdução do leite não materno, foram, respectivamente, 76, 120 e 176 dias. As razões de hazards foram 0,73 ( $\left.\mathrm{IC}_{95 \%}: 0,57-0,94\right)$ e 0,43 $\left(\mathrm{IC}_{95 \%}: 0,26-0,72\right)$, indicando que a introdução mais tardia do leite não materno é fator estatisticamente significativo para o prolongamento da amamentação. O teste de Cox da variável número de dias após a introdução de outro leite apresenta razão de hazards 0,9969 ( IC $_{95 \%}: 0,9950-0,9988$ ), com p $<0,01$.

\section{Discussão}

Nos primeiros meses de vida da criança, o preenchimento diário da planilha realizado pela mãe possivelmente foi mais minucioso devido à alimentação da criança ser pouco diversificada e das entrevistas domiciliares serem mais próximas, incentivando uma maior participação das mães. Após os seis meses de idade, é mais freqüente a criança ficar aos cuidados de outras pessoas e a introdução de alimentos é intensificada. Junto a isso, as entrevistas domiciliares passaram a ter intervalos maiores, observando-se menor participação espontânea da mãe. Nestes casos, o entrevistador, de maneira retrospectiva, coletava os dados de introdução dos alimentos e com que freqüência semanal eles foram oferecidos à criança até aquela data. Como o leite, mesmo após os seis meses de idade, é o alimento mais valorizado no primeiro ano de vida da criança ${ }^{19}$, supõe-se que, mesmo quando resgatada de modo retrospectivo, a idade de introdução do outro tipo de leite foi muito próxima da real.

Na interpretação da queda da porcentagem de crianças que ainda não receberam outro leite que não o materno, deve-se lembrar que a técnica de análise de sobrevida

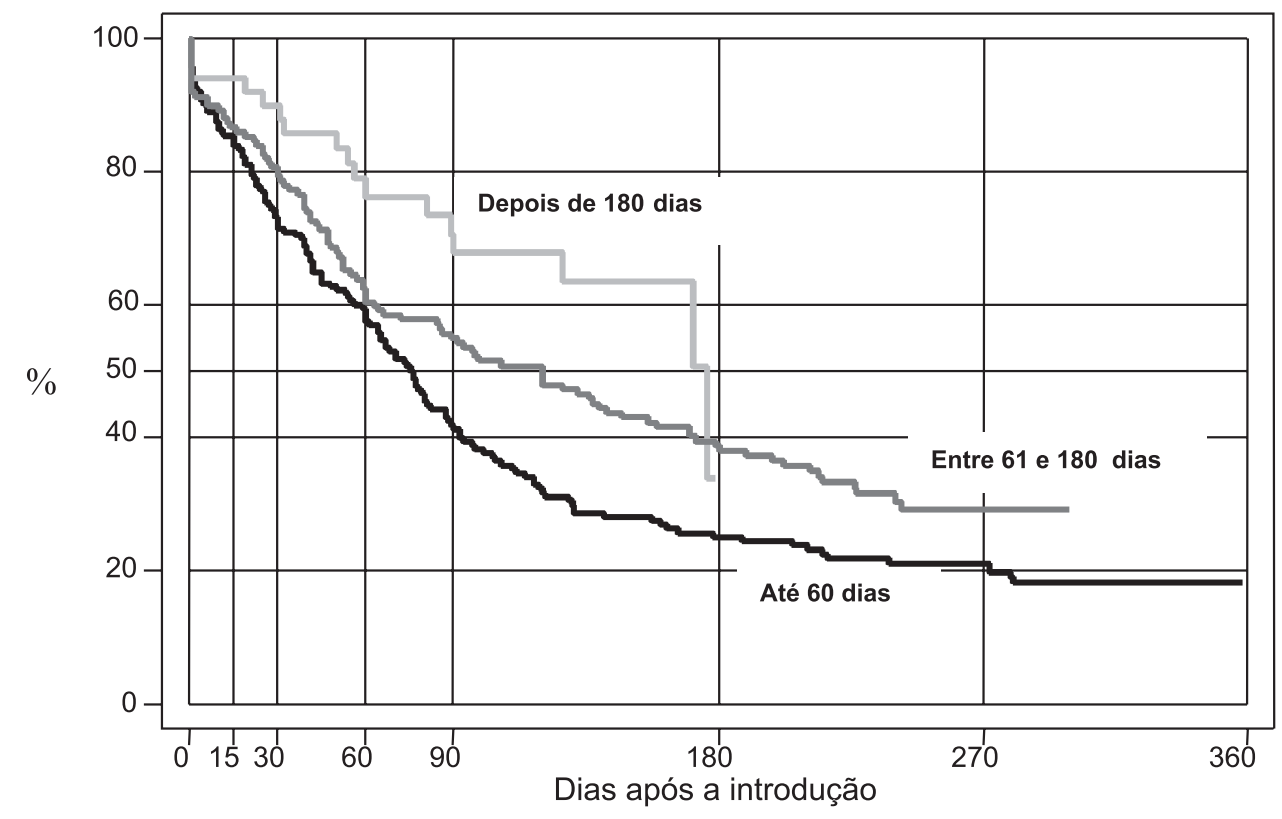

Figura 3 - Duração da amamentação em dias a partir da introdução de leite não materno, segundo a idade da criança quando da introdução.

Figure 3 - Duration of breastfeeding from the introduction of non-maternal milk, according to the child's age of introduction. 
ajusta os resultados, levando em consideração as perdas de observação (dados censurados). Admitindo-se que o leite de vaca, fluido ou em pó, formulado ou não, é um competidor do leite materno, e que sua introdução marca o início do processo de desmame, tem-se que este processo já começa aos dois meses de idade para metade das crianças. Alguns pesquisadores, ao estudarem a prática alimentar de crianças no primeiro ano de vida, também verificaram introdução precoce de alimentos, principalmente do leite ${ }^{19-21}$.

Na Figura 2, a variável tempo foi definida como a diferença "idade do fim da amamentação - idade de introdução do leite não materno", registrada pela mãe no questionário. Assim, uma diferença de 35 dias, por exemplo, pode significar introdução do outro leite aos 20 dias e abandono da amamentação aos 55 dias ou introdução aos 80 dias e abandono aos 115 dias, ou outras combinações possíveis. A Figura 3 procura amenizar este problema, apresentando curvas de desmame segundo épocas selecionadas de introdução de leite não materno; reconhece-se que dentro de cada período a dificuldade persiste, e que se está considerando as categorias como definidas no instante inicial da observação.

Parece lícito inferir que, quanto mais tarde é introduzido o outro leite, por mais tempo a mãe tende a amamentar seu filho após sua introdução, apesar de haver menos tempo disponível para o desmame acontecer, devido ao avanço da idade da criança, e se supor que, a partir de um ano, é alta a probabilidade do desmame total ocorrer. A in- trodução de outro leite depois que a amamentação está bem consolidada-como é o caso de crianças mais velhas - parece ter menor influência na diminuição da duração do aleitamento materno.

O presente resultado é concordante com o de Marques et al.22, que em estudo longitudinal com crianças do nordeste do Brasil, observaram idade mediana de introdução de outro leite de 24 dias e mediana de duração do aleitamento materno de 65 dias para crianças que iniciaram o consumo de leite antes do primeiro mês de vida, e de 165 dias para as demais, concluindo que a introdução precoce de outro leite está fortemente relacionada com a interrupção precoce do aleitamento materno.

Talvez um dos componentes no processo de desmame seja a compreensão da importância da amamentação pela mãe; mães desejosas de prolongar a amamentação o fazem retardando o mais possível a introdução de outro leite e, após a introdução, mantendo o maior tempo possível sua concomitância com o leite materno. Nas Figuras 2 e 3 não foram incluídas as 54 crianças que, durante o tempo de observação, nunca chegaram a receber outro leite. A inclusão destas crianças no grupo 3 englobaria as duas situações acima e reforçaria a argumentação, pois a nova curva, na Figura 3, ficaria ainda mais acima das outras duas.

Estudos com abordagem qualitativa podem trazer explicações sobre a atitude de introduzir precocemente outro leite e auxiliar nas propostas de estratégias mais eficazes para promoção, proteção e incentivo ao aleitamento materno.

\section{Referências}

1. World Health Organization. The optimal duration of exclusive breastfeeding: results of a WHO systematic review. Indian pediatr 2001; 38: 565-7.

2. Giugliani ER \& Victora CG. Alimentação complementar. J pediatr 2000; 76 (Suppl 3): S253-S262.
3. Hutlly SRA, Morris SS, Pisani V. Prevention of diarrhoea in young children in developing countries. Bull World Health Organ 1997; 75:163-74.

4. Wasmuth HE, Kolb H. Cow's milk and immunemediated diabetes. Proc Nutr Soc 2000; 59(4): 573-9. 
5. Gimeno SG, Souza JM. IDDM and milk consumption. A case-control study in São Paulo, Brazil. Diabetes Care 1997; 20(8): 1256-60.

6. Verge CF, Howard NJ, Irwig L, Simpson JM, Mackerras D, Silink M. Environmental factors in childhood IDDM. A population-based, case-control study. Diabetes Care 1994; 17(12): 1381-9.

7. Gillman MW, Rifas-Shiman SL, Camargo CA, Berkey CS, Frazier AL, Rockett HR et al. Risk of overweight among adolescents who wera breastfed as infants. JAMA 2001; 285(19): 2461-7.

8. Oddy WH, Holt PG, Sly PD, Read AW, Landau LI, Stanley FJ et al. Association between brestafeeding and asthma in 6 year old children: findings of a prospective birth cohort study. Br Med J 1999; 319(7213): 815-9.

9. Martin EM, Pascual CY, Garcia AMC. Prevention of food allergy. Allergol Immunopathol 1998; 26(3): 97101.

10. Forman MR. Review research on the factors associated with choice and duration of infant feeding in lessdeveloped countries. Pediatrics 1984; 74 (Suppl 4): 667-94.

11. Howie PW; McNeilly AS; Houston MJ; Cook A; Boyle H. Effect of supplementary food on suckling patterns and ovarian activity during lactation. Br Med J 1981; 283: $757-9$.
12. Hill PD; Humenick SS; Brennan ML; Woolley D. Does early supplementation affect long-term breastfeeding?. Clin Pediatr 1997; 36: 345-50.

13. Hörnell A; Hofvander Y, Kylberg E. Solids and formula: association with pattern and duration of breaastfeeding. Pediatrics 2001; (107)3: e38.

14. Rea M. Substitutos do leite humano - passado e presente. Rev Saúde Pública 1990; 24: 242-9.

15. Monteiro CA. Velhos e novos males da saúde no Brasil: a evolução do país e de suas doenças. São Paulo: HUCITEC/ABRASCO/NUPENS;1995.

16. Venancio SI, Monteiro CA. A tendência da prática da amamentaçäo no Brasil nas décadas de 70 e 80 . Rev Bras Epidemiol 1998; 1(1):40-9.

17. Souza, SB; Szarfarc, SC \& Souza, JMP. Prática alimentar no primeiro ano de vida em crianças atendidas em centros de saúde escola do Município de São Paulo. Rev Nutr 1999; 12: 167-74.

18. Klein, JP \& Moeschberger, ML. Survival analysis; techniques for censored and truncated data. New York: Springer; 1997.

Recebido em 23/07/01; aprovado em 17/06/02 